\title{
A Finite Element Model for the Simulation of Lost Foam Casting
}

\author{
G. Houzeaux and R. Codina
}

\begin{abstract}
In this paper, we present a numerical model to simulate lost foam casting processes. We first introduce this particular casting in order to catch the different physical processes in play during a casting. We also briefly comment on the possible physical and numerical models to envisage the numerical simulation. Next we present a model which aims at solving "part of" the complexities of the casting, together with a simple energy budget that enables to obtain an equation for the velocity of the metal front advance. Once the physical model is established we develop a finite element method to solve the governing equations. The numerical and physical methodologies are then validated through the solution of a two and a three-dimensional example. Finally, we briefly discuss some possible improvements of the numerical model in order to catch more physical phenomena.
\end{abstract}

\section{Contents}

1 Introduction $\quad 2$

2 Physical model $\quad 3$

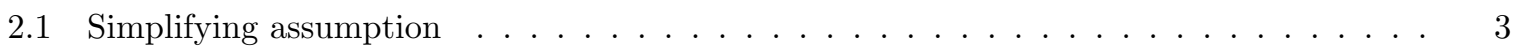

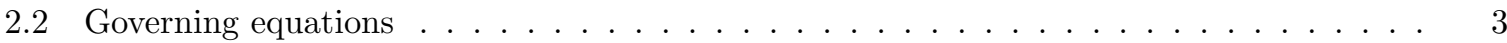

2.3 Front velocity . . . . . . . . . . . . . . . . . . . . . . . 4

2.4 Front tracking . . . . . . . . . . . . . . . . . . . . . . . . 5

2.5 Turbulence model . . . . . . . . . . . . . . . . . . . . . . . . . 6

2.6 Wall function approach .......................... 7

3 Numerical model $\quad 8$

3.1 Finite element method and time discretization . . . . . . . . . . . . . . . 8

3.2 Front advance treatment . . . . . . . . . . . . . . . . . . . . . 8 8

3.2 .1 Front definition . . . . . . . . . . . . . . ..... 8

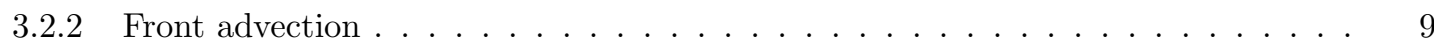

3.2.3 Further corrections ......................... 9

3.3 ALE method with fixed mesh . . . . . . . . . . . . . . . . . . . . . . 10

3.4 Numerical algorithm . . . . . . . . . . . . . . . . . . . . . . . . 11

4 Numerical examples $\quad 11$

4.1 Horizontal plate . . . . . . . . . . . . . . . . . . . . . . . 11

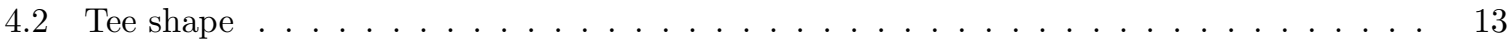

5 Conclusions $\quad 14$ 


\section{Introduction}

In this paper we present a numerical model for the simulation of a relatively new casting process, known as Lost Foam Casting (LFC) or Evaporative Pattern Casting (EPC). See [1] for an introduction to LFC. This process was developed during the sixties and has known an exponential growth since then. To have an idea of the importance gained by this technology in the last years, let us just mention that around $20 \%$ of the total aluminum casting in the US is obtained by LFC [2]. Despite its important place on the casting market, only recently numerical codes have allowed the numerical simulation of LFC. This is partly due to the complexity of the physical processes in play.

In general castings, known as Gravity Casting, the molten metal is poured into a mold filled with air. The metal is allowed to fill the mold thanks to gravity, as long as the pressure at the inflow is sufficiently high. In LFC, the air is replaced by expandable polystyrene (EPS). The polystyrene bead is placed in the mold, it is heated and expands until it completely fills the mold. The resulting cluster is dipped into a permeable refractory coating (of alumina, silica e.g.) which will act as a barrier between the sand and the metal, providing a control on the gases escape and a better surface finish. It is then embedded in a container filled with compacted sand and provided with a gating. See the sketch in Figure 1 (Left). LFC casting operates as follows. The molten metal is poured into the gating and once it gets in contact
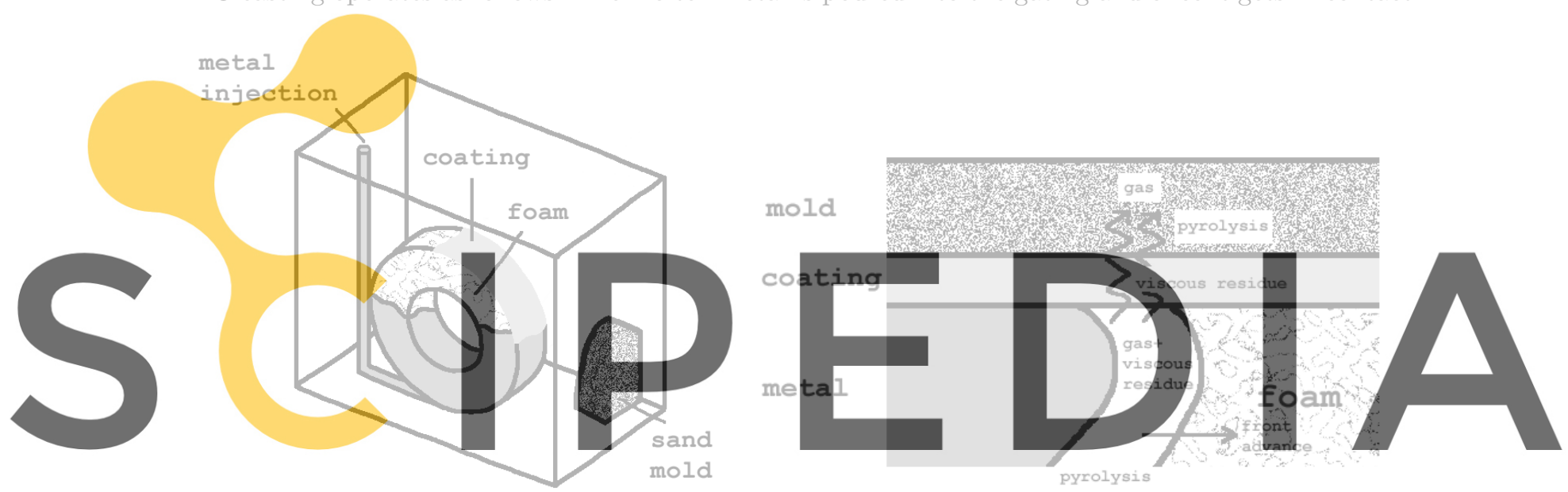

Register for free at https//www.scipedia.com to download the version without the watermark Figure 1: LFC. (Left) Main components. (Right) Main physical processes in play.

with the foam, the foam evaporates: this process is known as pyrolysis. Gases can vent through the sand if the coating is sufficiently premeable. However, if the gas is not vented sufficiently fast, casting defects can appear and perturb the metal advance. Figure 1 (Right) shows a simplified scheme of a casting using the lost foam technique. The main parameters that control the process are mainly the EPS pattern and combustion characteristics, the coating material, thickness, texture and permeability.

The numerical approach to LFC casting requires special attention at the physical as well as the numerical level. Due to the complex physical mechanisms involved, some physical simplifications are needed. For example, we decide not to try solving for the combustion of the foam. Instead, we obtain an equation for the metal front velocity, through a simple energy budget. Neither we consider the coating, although its effects can be taken into account into the equation for the front velocity. The foam is assumed to be burnt instantaneously, and the possible generation of viscous residues or defects is not considered [3]. The non-exhaustive list of the main assumptions is given in the first section. LFC usually involves characteristic velocities lower than that encountered in classical casting, and the metal flow can be either laminar or turbulent. We have chosen a turbulence model able to predict transition from laminar to turbulent state, namely the Spalart-Allmaras (SA) turbulence model which consists of one additional partial differential equation. However, in the examples presented in Section 3, the flows are considered fully turbulent and transition is not envisaged.

The numerical approximation chosen to solve the present problem is the finite element method. In order 
to capture the ever changing computational domain occupied by the metal, three main approaches are available [4]. The first method consists in writting the governing equations in a Lagrangian frame of reference, the nodes being advected by the flow velocity $[5,6]$. The second method is based on an Eulerian formulation. The position of the front is identified by a level set function [7], which is transported by the velocity $[8,9]$. The third method is the Arbitrary Lagrangian Eulerian (ALE) method, which uses both Lagrangian and Eulerian approaches, and consists in adapting the mesh to each new configuration but in limited zones; generally this implies a node movement near the front and a remeshing if the resulting mesh is too distorted $[10,11]$. Let us mention finally the promising meshless method [12] to solve free surface flows using a Lagrangian formulation. In this work we have chosen an ALE method, but with the originality that it is applied to a fixed mesh (FMALE). It will be described in Section 3.3.

\section{Physical model}

\subsection{Simplifying assumption}

We described in the first section the complexity of the physical processes involved in LFC. In order to treat LFC numerically, some simplyfying assumptions are needed. In addition, assumptions are added for the sake of clarity of the exposition. They are:

1. Metal and foam properties are constant (at least locally).

2. There is no solidification during filling.

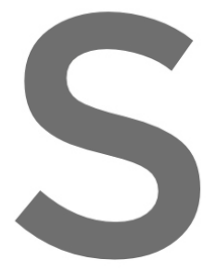

3. There is no source of contaminant, like viscous residues. A simple equation to take into account 4he generation of contaminants will be given in Appendix B.
for the foam is, in principle, needed.
5. All the combustion gases escape through the coating and the mold. Therefore, no gas is trapped
between the metal and the foam.

According to these simplifications, we are able to devise a concise physical model, presented in the Register for free aring https//www.scipedia.com to download the version without the watermark

\subsection{Governing equations}

Let $\Omega$ be the domain of the mold, $\Omega_{\mathrm{m}}$ the domain that is filled by the molten metal and $\Omega_{\mathrm{f}}$ the domain occupied by the foam. They are shown in Figure 2. Obviously, both domains depend on time.

Let us denote the physical properties as follows: $\rho$ as the density, $\nu$ as the kinematic viscosity, $c_{p}$ as the specific heat at constant pressure, $\kappa$ as the thermal diffusion coefficient and $\alpha_{\mathrm{ij}}$ as the heat transfer coefficient between materials $\mathrm{i}$ and $\mathrm{j}$. We introduce the kinematic pressure $p=P / \rho$ where $P$ is the mechanical pressure. The unknowns to be determined in the general case are $\boldsymbol{u}$ the velocity, $p$ the pressure and $T$ the temperature of the molten metal. Let us denote $u_{\mathrm{mf}}$ as the velocity at which the front of molten metal advances through the foam.

The subscripts $\mathrm{m}$, f and o will be used to refer to molten metal, foam and mold, respectively, for the physical properties. Likewise, $\Gamma_{\mathrm{ij}}$ will be used to denote the interface between materials $\mathrm{i}$ and $\mathrm{j}$, and subscript inf will refer to values at the inflow of the domain; see Figure 2.

The equations describing the lost foam model in an Eulerian frame of reference are the following:

$$
\begin{aligned}
\partial_{t} \boldsymbol{u}+\left(\boldsymbol{u}_{a} \cdot \nabla\right) \boldsymbol{u}-2 \nabla \cdot\left[\nu_{\mathrm{m}} \varepsilon(\boldsymbol{u})\right]+\nabla p & =0, \\
\nabla \cdot \boldsymbol{u} & =0, \\
\partial_{t} T+\boldsymbol{u}_{a} \cdot \nabla T-\nabla \cdot\left(\kappa_{\mathrm{m}} \nabla T\right) & =0,
\end{aligned}
$$




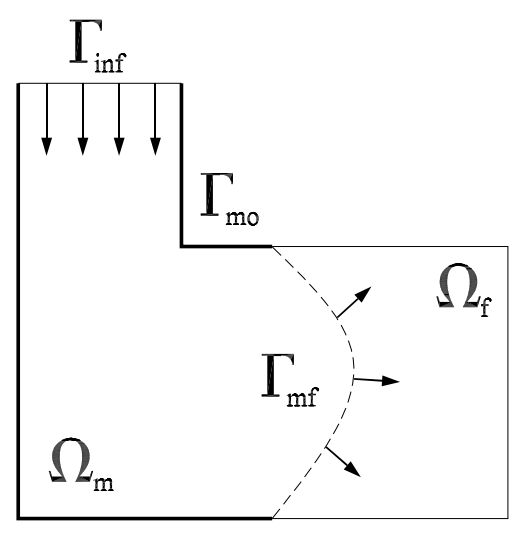

Figure 2: Domain and boundary notation.

to be solved in $\Omega_{\mathrm{m}}$, where $\varepsilon(u)$ is the rate of deformation tensor given by

$$
\varepsilon(u)=\frac{1}{2}\left(\nabla u+\nabla u^{t}\right) .
$$

We note that the advection velocity of the momentum and heat equations should be corrected from $u$ to $\boldsymbol{u}_{a}$ as the domain $\Omega_{\mathrm{m}}$ is moving; this point will be treated in Section 3.3. Likewise, the kinematic viscosity $\nu_{\mathrm{m}}$ and the diffusivity $\kappa_{\mathrm{m}}$ could be modified if a turbulence model is used. The governing set of equations must be provided with initial and boundary conditions, the latterer one being:
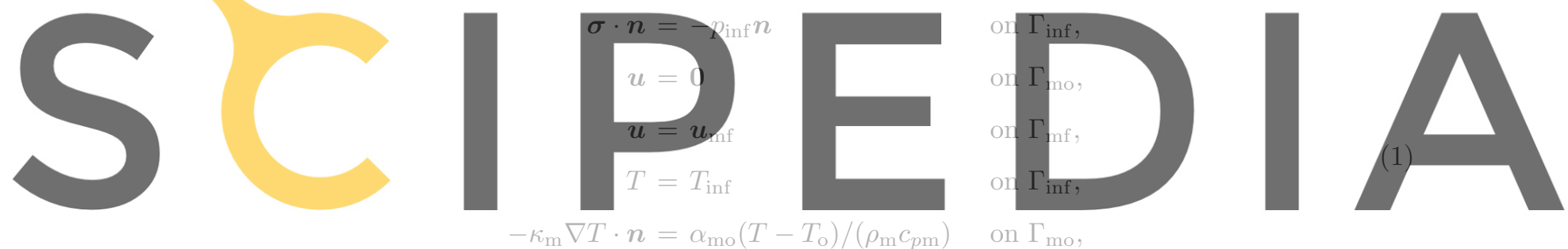

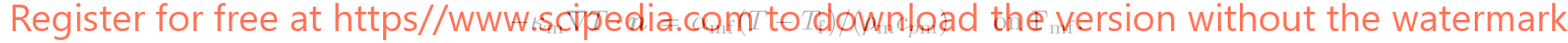

The vector $n$ is the outward unit normal to domain $\Omega_{\mathrm{m}}$, the tensor $\sigma$ is the stress tensor given by

$$
\boldsymbol{\sigma}=-p \boldsymbol{I}+2 \nu_{\mathrm{m}} \boldsymbol{\varepsilon}(\boldsymbol{u})
$$

$\boldsymbol{I}$ being the $n_{\mathrm{d}}$-dimensional identity. $T_{\mathrm{inf}}$ is the pouring temperature and $p_{\text {inf }}$ is the pouring pressure. We note that the boundary condition given by Equation (1) 1 at the inflow is

$$
p=p_{\text {inf }} \quad \text { on } \Gamma_{\text {inf }},
$$

only if $\varepsilon(\boldsymbol{u})=0$, which is satisfied, for example, if the flow is fully developed.

In the governing set of equations, apart from the velocity, pressure and temperature, we are left with two unknowns, namely the front advance velocity and the time dependent domain $\Omega_{\mathrm{m}}$. A model for the front advance velocity is now given. Next a front advance tracking is presented to tackle the evolution of $\Omega_{\mathrm{m}}$. Finally, to close the physical description of the problem, we end up with the description of the turbulence model and the associated treatment for the wall boundary conditions.

\subsection{Front velocity}

The front velocity at which the front advances is a function of the heat absorbed by the foam, which in turn depends on $\boldsymbol{u}$ and $T$ so that $u_{\mathrm{mf}}=u_{\mathrm{mf}}(\boldsymbol{u}, T)$. If it is high enough, the foam will be molten and 
vaporized, and the room left free will be occupied by the metal. The front velocity can be computed from a simple global balance of energy. Suppose that a point of the interface molten metal-foam advances a distance $\delta s$ normal to the surface of the interface in a time $\delta t$. The heat per unit surface released from the molten metal to the volume initially occupied by foam will be

$$
Q_{\text {rel }}=\alpha_{\mathrm{mf}}\left(T_{\mathrm{m}}-T_{\mathrm{f}}\right) \delta t .
$$

This heat will be invested in melting and vaporazing the foam and in increasing the temperature of the spatial points, which originally are occupied by foam and at the end of the process are filled with metal. Therefore, the heat stored can be approximated by

$$
Q_{\text {sto }}=\left[\rho_{\mathrm{f}} c_{p \mathrm{f}} \frac{1}{\delta t}\left(T_{\mathrm{m}}-T_{\mathrm{f}}\right)+\frac{\rho_{\mathrm{f}}}{\delta t}\left(E_{\mathrm{mel}}+E_{\mathrm{vap}}\right)\right] \delta s \delta t,
$$

where $E_{\text {mel }}$ and $E_{\text {vap }}$ are the melting and vaporization energy, respectively, which must be determined from experiments. Imposing that $Q_{\text {sto }}=Q_{\text {rel }}$ it is found that

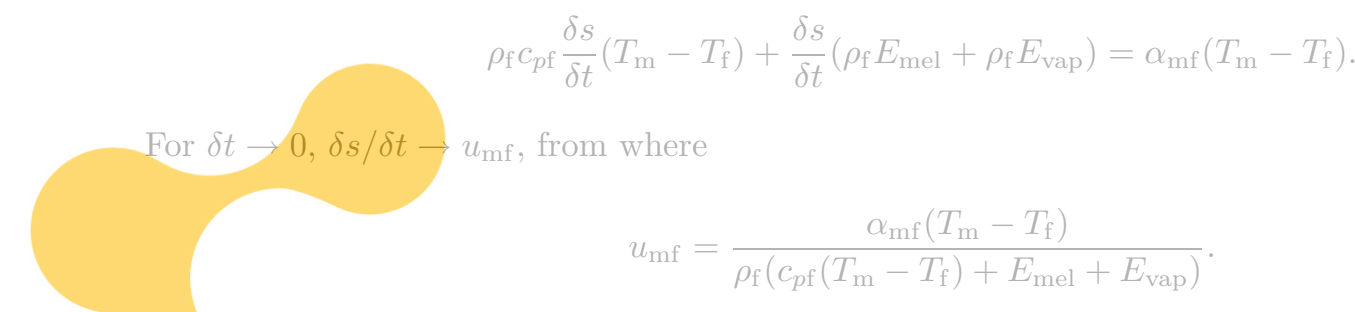

In Appendix C, we will present a front velocity model that takes into account the possible effects of the

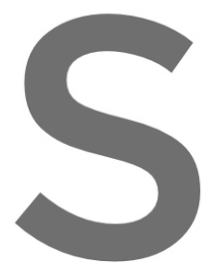

back-pressure exerted

2.4 Front tracking

One possibility of defining the molten metal-foam
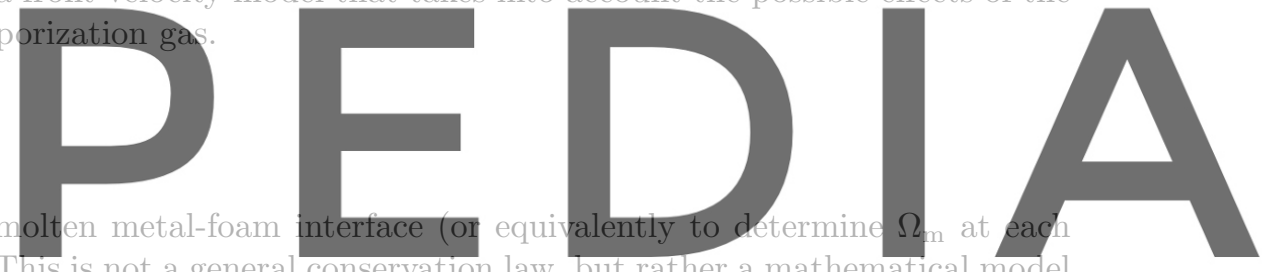

to describe the evolution of this interface (see [8] for an application of this method to classical mould

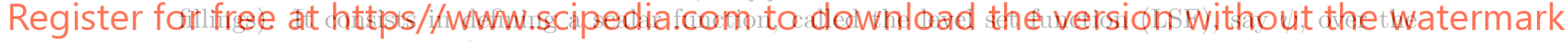

computational domain $\Omega$ in such a manner that its value at a certain point indicates the presence or absence of metal. This function may be considered a fictitious fluid property. For instance, we may assign the value 1 to regions where the liquid metal has already entered and the value 0 to foam-filled regions. The position of the metal front will be defined by the isovalue contour $\psi=\psi_{c}$ where $\psi_{c} \in[0,1]$ is a critical value defined a priori. We usually take $\psi_{c}=0.5$. This value is immaterial if $\psi$ is a true step function, but is needed in the finite element discretization. The level set technique is usually used to follow free surfaces of fluids, i.e. surfaces advected by the fluid velocity itself. In the present case we have a somewhat different situation. The LSF is advected due to temperature gradients and is therefore not explicitly advected by the fluid motion (Equation (2)). The fluid motion is induced by the front advance and we can define formally:

$$
\Omega_{\mathrm{m}}=\left\{\boldsymbol{x} \in \Omega \mid \psi(\boldsymbol{x}) \geq \psi_{c}\right\}
$$

In order to follow the motion of the front, a level set equation is solved in the following way:

$$
\frac{\partial \psi}{\partial t}+\boldsymbol{u} \cdot \nabla \psi=0
$$

where $\boldsymbol{u}$ is the velocity of the metal. Remember that at the metal-foam interface, the velocity is imposed as a Dirichlet boundary condition given by Equation (1) 3. The algorithm that allows us to correctly advect the LSF will be presented in Section 3.2, as its implementation is completely dependent on the 
numerical strategy presented in Section 3, namely the finite element method. Equation (3) must be provided with initial and boundary conditions. For example:

$$
\psi=1 \quad \text { on } \Gamma_{\text {inf }} .
$$

In the latter section we derived an equation for the front velocity module. We now assume that the front is moving along the normal to the front, which is precisely the gradient of the level set function:

$$
\boldsymbol{u}_{\mathrm{mf}}=-\frac{\nabla \psi}{|\nabla \psi|} u_{\mathrm{mf}}
$$

\subsection{Turbulence model}

Turbulence is treated using the ensemble-averaging approach together with the Boussinesq approximation for the Reynolds stress tensor, and a similar apparent diffusion concept for the heat equation. The resulting system is referred to as Reynolds-averaged Navier-Stokes (RANS) equations and reads:

$$
\begin{aligned}
\partial_{t} \boldsymbol{u}+\left(\boldsymbol{u}_{a} \cdot \nabla\right) \boldsymbol{u}-2 \nabla \cdot\left[\left(\nu_{\mathrm{m}}+\nu_{t}\right) \varepsilon(\boldsymbol{u})\right]+\nabla p & =0, \\
\nabla \cdot \boldsymbol{u} & =0, \\
\partial_{t} T+\boldsymbol{u}_{a} \cdot \nabla T-\nabla \cdot\left[\left(\kappa_{\mathrm{m}}+\kappa_{t}\right) \nabla T\right] & =0,
\end{aligned}
$$

where $\nu_{t}$ is the eddy viscosity (also referred to as apparent or turbulent viscosity), and $\kappa_{t}$ is the turbulent diffusivity.
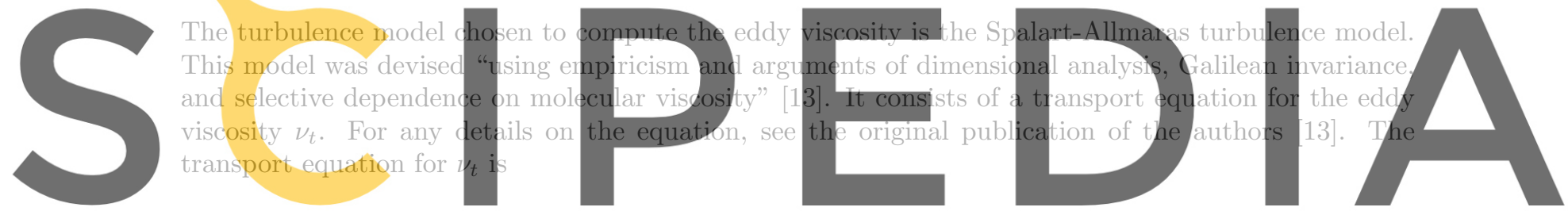

$$
\partial_{t} \nu_{t}+u \cdot \nabla \nu_{t}-c_{b_{1}} S \nu_{t}-\frac{1}{2}\left[\nabla \cdot\left(\nu_{t} \nabla \nu_{t}\right)+c_{b_{2}}\left(\nabla \nu_{t}\right)^{2}\right]+c_{w_{1}} f_{w} \frac{\nu_{t}^{2}}{l^{2}}=0
$$

Register for free at https//www.scipedia.com to download the version without the watermark

where $c_{b_{1}}, c_{b_{2}}, \sigma$ and $c_{w_{1}}$ are constants, $S$ is the norm of the vorticity, $f_{w}$ is a function depending on $S, \nu_{t}$ and the distance to the wall $d$. This equation is the high Reynolds number version of the model. Additional corrections enable for example to compute low Reynolds number and transition effects, not shown here for the sake of brevity.

The turbulent diffusivity is calculated through the introduction of the turbulent Prandtl number $\operatorname{Pr}_{t}$ defined as:

$$
\kappa_{t}=\frac{\nu_{t}}{\operatorname{Pr}_{t}}
$$

In order to close the system of governing equations, an expression for $\operatorname{Pr}_{t}$ is needed. In general, we have that:

$$
\text { For liquid metals: } \operatorname{Pr} \ll 1, \quad \operatorname{Pr}_{t}>1, \quad \operatorname{Pr}_{t}=\operatorname{Pr}_{t}(\operatorname{Pr}, \operatorname{Re}, y) \text {, }
$$

where Re is the Reynolds nymber and $y$ is the distance to the wall $[14,15]$. The turbulent Prandtl number increases with decreasing Prandtl and Reynolds numbers. In [16] the authors mention that both theory and experiment suggest that $\mathrm{Pr}_{t} \rightarrow \mathrm{Pr}_{t \infty}$, a value common to all $\mathrm{Pr}$, as $\operatorname{Re} \rightarrow \infty$. They derive an expression for the turbulent Prandtl number in pipe and channel flows with $\operatorname{Pr}_{t \infty}=0.85$. This is the value chosen in the present work. The system of governing equations is now closed. 


\subsection{Wall function approach}

The RANS and turbulence equations are solved using the wall function approach [17] on the wall-type boundaries of the computational domain, i.e. $\Gamma_{\text {mo }}$ in the present case. In order to avoid solving for the large gradients present in the boundary layer, the wall function approach implemented here consists in assuming that the computational wall is located at a distance $y$ sufficiently far from the real wall where the no-slip condition for the velocity holds. Then the wall friction $U_{*}$ is estimated applying the law of the wall at $y$, i.e. by solving the non-linear Reichardt's law given by (see for example [18]):

$$
U^{+}:=\frac{U}{U_{*}}=\frac{1}{\chi} \ln \left(1+\chi y^{+}\right)+7.8\left[1-\exp \left(-\frac{y^{+}}{11}\right)-\frac{y^{+}}{11} \exp \left(-0.33 y^{+}\right)\right],
$$

where $U$ is the velocity at the computational wall, $\chi=0.41$ is the Von Karman constant and where $y^{+}$ is the dimensionless distance to the wall of the boundary point defined as

$$
y^{+}:=\frac{y U_{*}}{\nu_{\mathrm{m}}} .
$$

The wall boundary condition for the momentum equations consists of a mixed Dirichlet/Neumann condition where the normal component of the velocity is zero and where the tangential component of the traction $\boldsymbol{t}=\sigma \cdot n-n \cdot \sigma \cdot n$ is given by

$$
t=-U_{*}^{2} \frac{u}{|u|}
$$
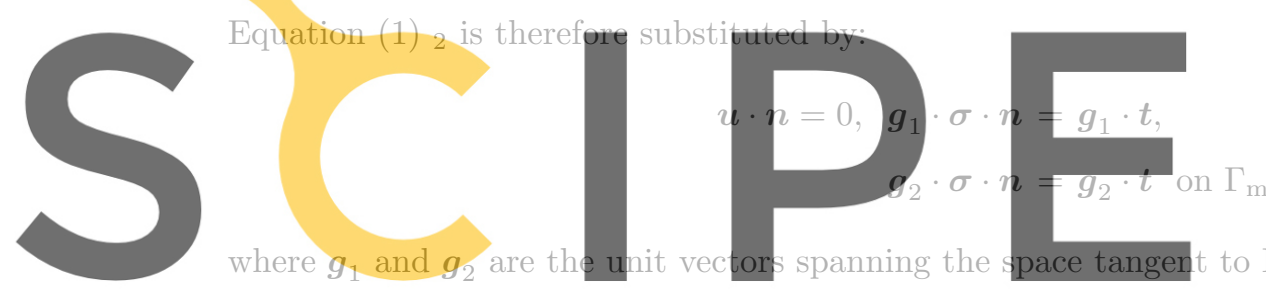

where $g_{1}$ and $g_{2}$ are the unit vectors spanning the space tangen
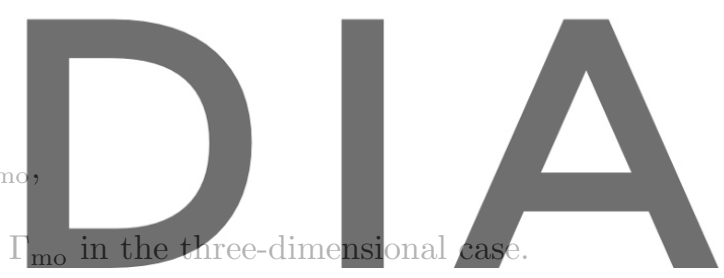

The wall condition for the eddy viscosity is computed using the classical mixing length hypothesis

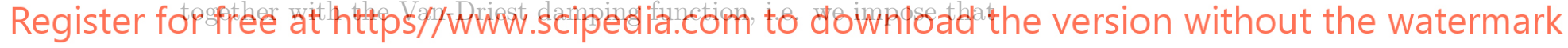

$$
\begin{aligned}
\nu_{t} & =l_{m i x}^{2}\left|\frac{d U}{d y}\right|, \\
l_{m i x} & =\chi y^{+}\left[1-\exp \left(-\frac{y^{+}}{26}\right)\right],
\end{aligned}
$$

where $\frac{d U}{d y}$ is the normal derivative of the tangential velocity. Equation (7) corresponds to the inner-layer equation of Baldwin-Lomax model, approximating the magnitude of the vorticity by $d U / d y$. It can be re-expressed in terms of the dimensionless quantities as:

$$
\nu_{t}^{+}=\chi^{2}\left(y^{+}\right)^{2}\left[1-\exp \left(-\frac{y^{+}}{26}\right)\right]^{2} \frac{d U^{+}}{d y^{+}}, \quad \nu_{t}^{+}=\frac{\nu_{t}}{\nu_{\mathrm{m}}}
$$

where $d U^{+} / d y^{+}$is obtained by simply deriving Reichardt's law (6), i.e.

$$
\frac{d U^{+}}{d y^{+}}=\frac{1}{1+\chi y^{+}}+\frac{7.8}{11}\left[\exp \left(\frac{-y^{+}}{11}\right)+\left(0.33 y^{+}-1\right) \exp \left(-0.33 y^{+}\right)\right]
$$

In the present work, we leave the wall boundary condition for the temperature unchanged although the heat transfer coefficient may be different in the turbulent case. 


\section{Numerical model}

In the previous section we presented the physical modeling of the lost foam process. However, some purely numerical ingredients are necessary to solve the governing equations efficiently. We first introduce the finite element method which is used to solve the partial differential equations. Next we introduce the treatment of the front advance. Then we explain how this method affects the governing equations; in particular we will see that it can be viewed as an arbitrary Lagrangian (ALE) method applied to fixed meshes. Then we explain the complete numerical algorithm.

\subsection{Finite element method and time discretization}

The RANS, heat and SA equations are solved using a Finite Element model based on a stabilized Galerkin method. In fact, it is well-known that the Galerkin formulation can lack stability for two major reasons. The first reason is related to the compatibility of the finite element spaces for the velocity and the pressure which have to satisfy the so-called Ladyzhenskaya-Brezzi-Babuška (LBB) condition. This condition is necessary to obtain a stability estimate for the pressure; without requiring this condition, the pressure would be out of control. The second reason is attributed to the relative importance of the viscous and convective effects in the momentum equation. The stabilized formulation is based on the algebraic variational subgrid scale (SGS) model first introduced in [19]. The variational SGS model uses as a starting argument that the inability of the mesh to resolve all the flow scales is responsible for the numerical instabilities. Therefore, the model calculates in some approximate way the unresolved scales of the flow, i.e. the scales smaller than the mesh size. The method is extensively described in [20]. Finally, the time discretization is carried out using the generalized trapezoidal rule, i.e. a finite difference scheme. To do so, let us consider a partition $0=t^{0}<t^{1}<\cdots<t^{N}=T$ of the

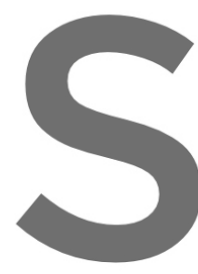
time interval $[0, T]$ of interest. T
In this work we consider two ty
and the pressure. The Q1/Q1 el
velocity and pressure. We will al
pressure. These elements do not A similar numerical mo
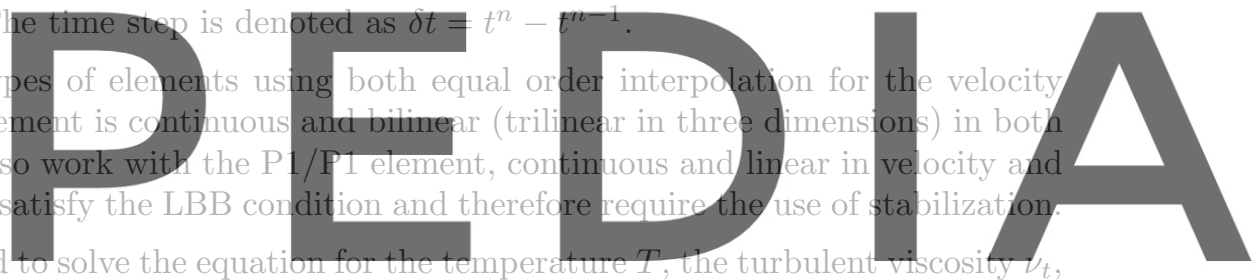

and the LSF $\psi$, which are interpolated like $u$ and $p$. They are integrated in time using the generalized

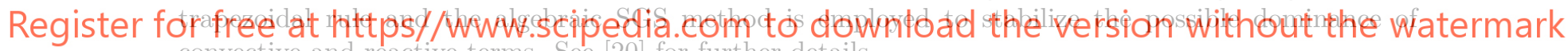
convective and reactive terms. See 20 for further details.

\subsection{Front advance treatment}

In Section 2.4, we mentioned the possibility of identifying the presence and absence of metal by solving a partial differential equation for the LSF. In this work, we propose to solve the partial differential equations in the whole domain $\Omega$, in order to advect the LSF correctly across the interface. We therefore have to define in a clear way the metal domain, the domain of interest. As presented here, the proposed method is exclusive to the finite element method (although it can surely be extended to other methods).

\subsubsection{Front definition}

Let us take as reference the solution obtained at a time $t^{n}$. We assume that the LSF $\psi$ is known. The idea is to divide the finite element mesh of $\Omega$ into three zones, which are three sets of elements of the finite element discretization: the metal set with elements whose nodes satisfy $\psi>\psi_{c}$; the foam set with elements whose nodes satisfy $\psi \leq \psi_{c}$; and finally, the front set which includes elements that have both metal and foam nodes, i.e. the remaining elements. In the same way, we define the front nodes as the nodes belonging to front elements, which in addition can be of metal or foam. Metal nodes are nodes of metal elements; likewise, we define foam nodes similarly. These metal and foam nodes can be of front type also. See Figure 3. 


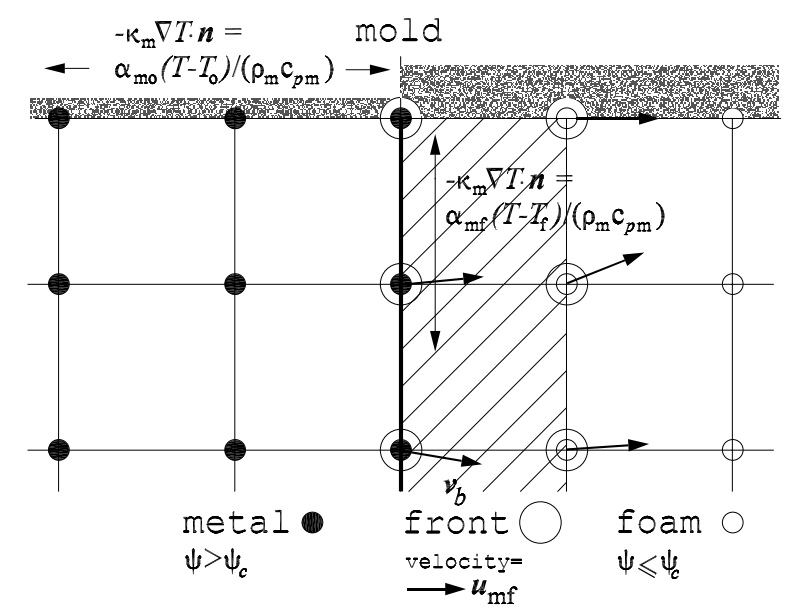

Figure 3: Scheme of the front treatment.

\subsubsection{Front advection}

The front velocity given by Equations (2) and (4) is imposed as a Dirichlet boundary condition on all the front nodes. This enables to advect the LSF properly across the metal-foam interface, both in the metal and foam domain. Here we must list some useful numerical ingredients:
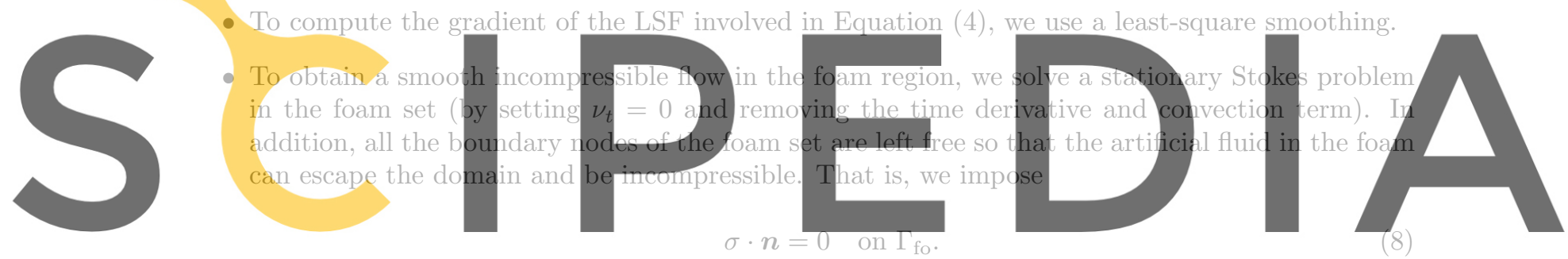

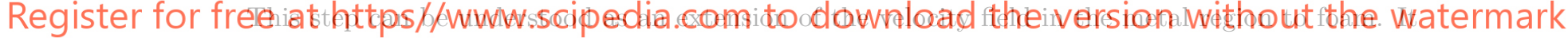 \\ is necessary for the numerical problem (not for the continuous one) since at each time step the front will enter several foam nodes where the velocity needs to be defined.}

- To insulate the metal set completely from the foam set, the element matrices of the front set are not assembled in the global stiffness matrix of the RANS, SA and heat equations. Note that for numerical reasons, it is preferable to multiply the front element matrices by a small value (say $10^{-6}$ ) rather than removing it from the assembly. What we have now are two independent domains, one filled with metal, the other filled with foam, and a front of one element width on which the velocity is prescribed.

- The velocity vectors of the front nodes located on the mold boundary are projected on the boundary so that the metal does not escape the metal domain as sketched in Figure 3.

\subsubsection{Further corrections}

As long as the heat equation is concerned, by removing the front element contribution to the stiffness matrix we are imposing implicitly an adiabatic condition for the metal domain, that is $\alpha_{\mathrm{mf}}=0$ in Equation $1_{6}$. However, if $\alpha_{\mathrm{mf}} \neq 0$, the adiabatic condition can be converted into the desired boundary condition by reconstructing the boundary. This is explained Appendix A at the end of the paper. In the independent foam domain, the temperature can be set constant. 


\subsection{ALE method with fixed mesh}

When using the level set technique in general casting, involving a metal-air mixture, the Navier-Stokes equations are solved in the entire mold, the physical properties being those of the metal or those of the air according to the value of $\psi$. The frame of reference is therefore Eulerian. In the present case, the LSF is used to define the metal computational domain, which changes at all time. However, according to the numerical algorithm used to capture the front advance, we want to use the same mesh all along the time integration. So how do we interpret the method described in last section? Figure 4 illustrates the method we propose together with the classical ALE method on a simple one-dimensional example, on the $x$-axis. The filled bullets represents metal nodes while the empty ones represent foam nodes. Filled

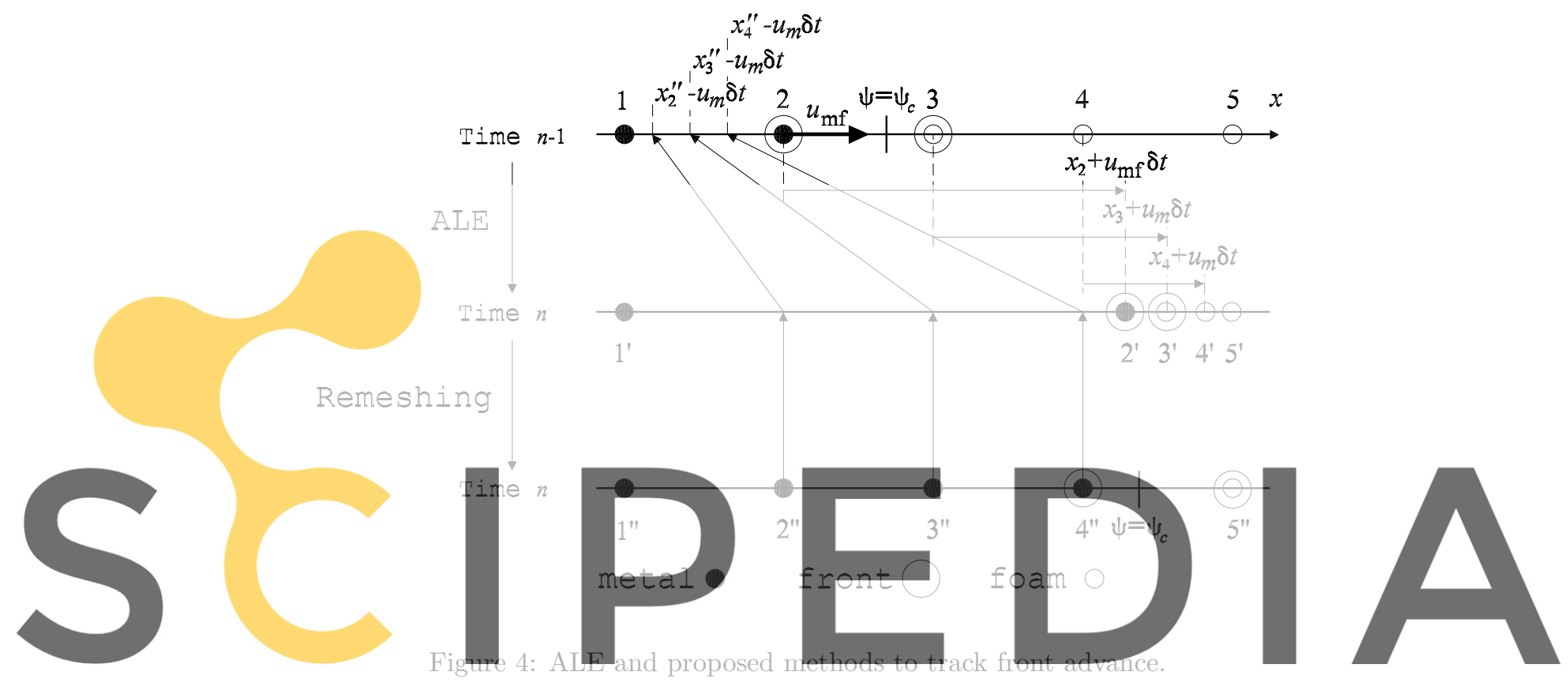

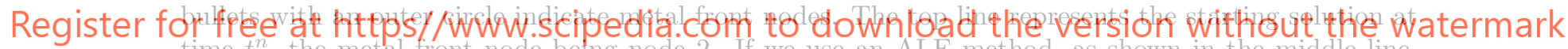

time $t^{n}$, the metal tront node being node 2. If we use an ALE method, as shown in the midale line

nodes 2,3 and 4 are moved with velocity $u_{m}=u_{m}(x)$. In particular, node 2 moves with a velocity

$u_{m}=u_{\mathrm{mf}}$. We recall that when the mesh is moving with velocity $\boldsymbol{u}_{m}$, the advection velocity $\boldsymbol{u}_{a}$ of the transport equations should be substituted by $\boldsymbol{u}-\boldsymbol{u}_{m}$. Now let us remesh the new mesh so that it coincides with the original mesh. The solution is sketched on the third line. We observe that going back to the original mesh, the nodes $2^{\prime \prime}, 3^{\prime \prime}$ and $4^{\prime \prime}$, with coordinates $x_{2^{\prime \prime}}-u_{m} \delta t, x_{3^{\prime \prime}}-u_{m} \delta t$ and $x_{4^{\prime \prime}}-u_{m} \delta t$, respectively would fall in the element with nodes 1 and 2 . If $u_{m}$ is known, we can therefore find the values of $\boldsymbol{u}, T$ and $\nu_{t}$ at time $n$ of the newly metalized nodes. We take for these nodes $u_{m}=u^{n-1}$. Once we know the position of nodes 1 ", 2 ", 3 " and 4" at time $t^{n}$, we can obtain the value of the unknowns by any interpolation procedure at hand. These values are those needed in the time discrete form of the differential equations. 


\subsection{Numerical algorithm}

We now sump up all the concepts introduced in the previous section. The partial differential equations to solve in $\Omega$ are:

$$
\begin{aligned}
\partial_{t} \boldsymbol{u}+\left[\left(\boldsymbol{u}-\boldsymbol{u}_{m}\right) \cdot \nabla\right] \boldsymbol{u}-2 \nabla \cdot\left[\left(\nu+\nu_{t}\right) \boldsymbol{\varepsilon}(\boldsymbol{u})\right]+\nabla p & =0, \\
\nabla \cdot \boldsymbol{u} & =0 \\
\partial_{t} \nu_{t}+\left(\boldsymbol{u}-\boldsymbol{u}_{m}\right) \cdot \nabla \nu_{t}-c_{b_{1}} S \nu_{t}-\frac{1}{\sigma}\left[\nabla \cdot\left(\nu_{t} \nabla \nu_{t}\right)+c_{b_{2}}\left(\nabla \nu_{t}\right)^{2}\right]+c_{w_{1}} f_{w} \frac{\nu_{t}{ }^{2}}{d^{2}} & =0 \\
\partial_{t} T+\left(\boldsymbol{u}-\boldsymbol{u}_{m}\right) \cdot \nabla T-\nabla \cdot\left[\left(\kappa+\kappa_{t}\right) \nabla T\right] & =0 \\
\frac{\partial \psi}{\partial t}+\boldsymbol{u} \cdot \nabla \psi & =0 .
\end{aligned}
$$

The turbulent diffusivity coefficient $\kappa_{t}$ is given by Equation (5). The physical properties $\nu$ and $\kappa$, the mesh velocity $\boldsymbol{u}_{m}$, and the boundary conditions change with time, according to the position of the front (identified by the value of $\psi$ ). Their calculations are presented in Algorithm 1.

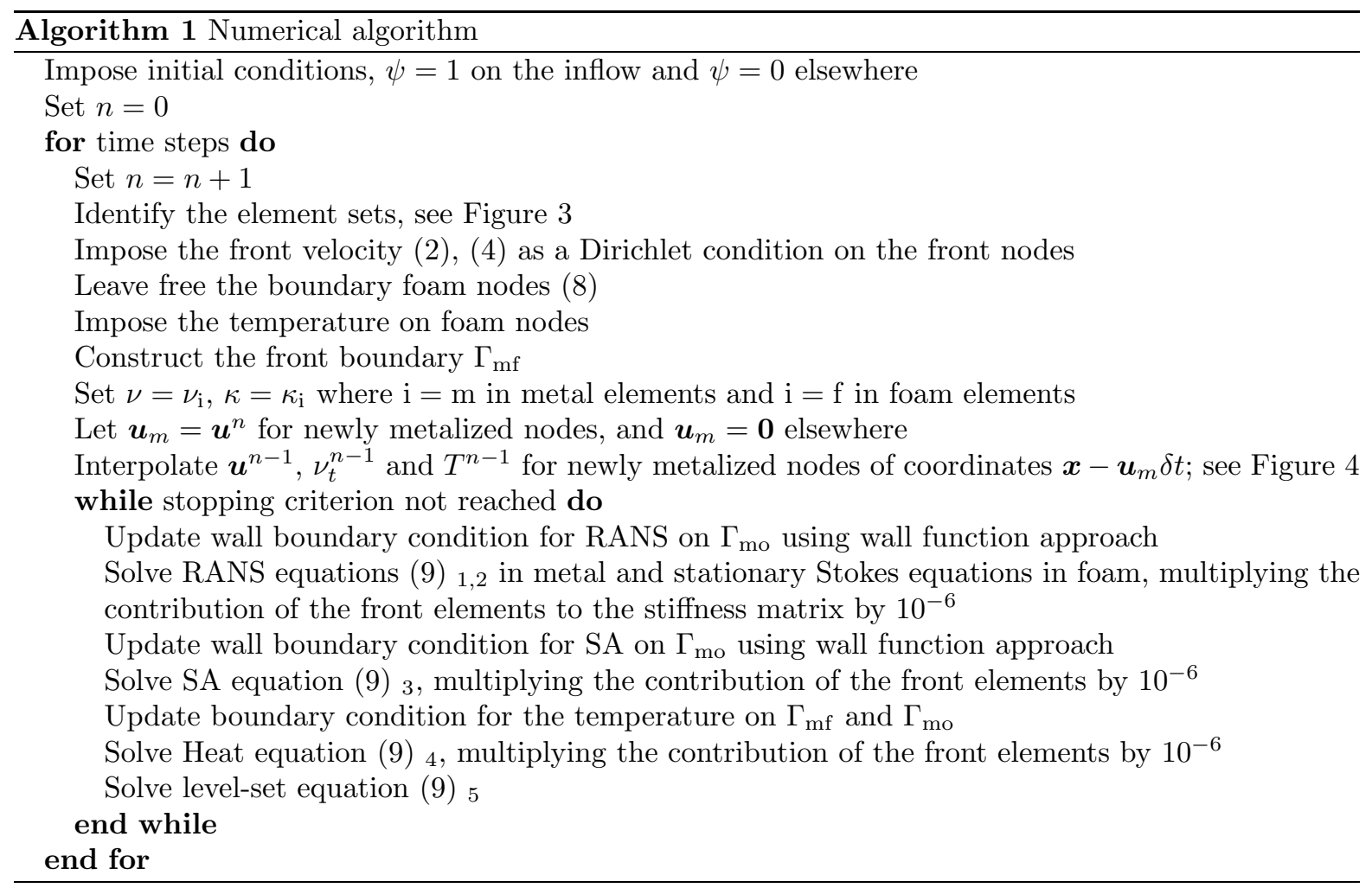

\section{Numerical examples}

\subsection{Horizontal plate}

The first example we solve is the aluminum casting of a two-dimensional plate [21, 3]. The geometry is shown in Figure 5. We consider that the properties of the material do not depend on the temperature. They are shown in Table 1. For the sake of simplicity, we set $\alpha_{\mathrm{mo}}=0$ and $\alpha_{\mathrm{mf}}=0$. Note that the Prandtl number of this liquid metal is very small, $\operatorname{Pr}=0.0045$. This means that the diffusion of the 


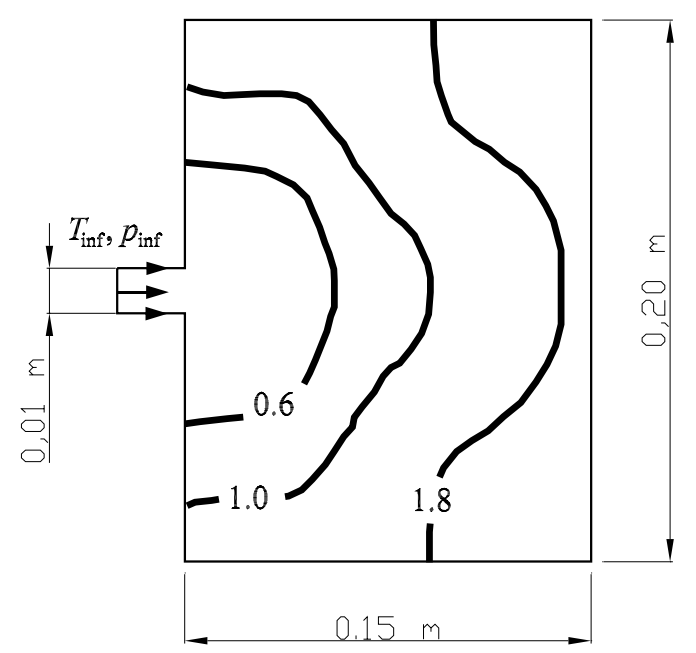

Figure 5: Horizontal plate. Geometry, boundary conditions and experimental front advance [21].

temperature is much more efficient than the diffusion of momentum, and therefore the boundary layer of the temperature is much larger than that of the velocity. Through the solution of this example we want

\begin{tabular}{llll}
\hline \multicolumn{3}{l}{ Symbol } & Palue \\
\hline \multicolumn{2}{l}{ Aluminum } & \\
$\alpha_{\mathrm{mf}}$ & Metal to foam heat transfer coefficient & $6.00 \times 10^{3}$ & {$\left[\mathrm{~kg} /\left(\mathrm{s}^{3} \mathrm{~K}\right)\right]$} \\
$\rho_{\mathrm{m}}$ & Density & $2.70 \times 10^{3}$ & {$\left[\mathrm{~kg} /\left(\mathrm{m}^{3}\right)\right]$} \\
$\nu_{\mathrm{m}}$ & Viscosity & $3.70 \times 10^{-7}$ & {$\left[\mathrm{~m}^{2} / \mathrm{s}\right]$} \\
$\kappa_{\mathrm{m}}$ & Conductivity & $8.32 \times 10^{-5}$ & {$\left[\mathrm{~m}^{2} / \mathrm{s}\right]$} \\
$c_{p \mathrm{~m}}$ & Specific heat & $8.37 \times 10^{2}$ & {$[\mathrm{~J} /(\mathrm{kg} \mathrm{K})]$} \\
$T_{\mathrm{inf}}$ & Inflow temperature & $1.02 \times 10^{3}$ & {$[\mathrm{~K}]$} \\
\hline Foam & & \\
$\rho_{\mathrm{f}}$ & Density & $2.00 \times 10^{1}$ & {$\left[\mathrm{~kg} / \mathrm{m}^{3}\right]$} \\
$c_{p \mathrm{f}}$ & Specific heat & $4.00 \times 10^{3}$ & {$[\mathrm{~J} /(\mathrm{kg} \mathrm{K})]$} \\
$E_{\mathrm{mel}}$ & Specific melting heat & $8.00 \times 10^{2}$ & {$[\mathrm{~J} / \mathrm{kg}]$} \\
$E_{\mathrm{vap}}$ & Specific vaporization heat & $4.00 \times 10^{2}$ & {$[\mathrm{~J} / \mathrm{kg}]$} \\
$T_{\mathrm{f}}$ & Temperature & $2.93 \times 10^{2}$ & {$[\mathrm{~K}]$} \\
\hline
\end{tabular}

Table 1: Horizontal plate. Physical properties and variables in play.

to test the numerical strategy. We consider the following numerical/physical combinations: laminar and turbulent flows; a fine mesh of 3660 Q1/Q1 elements and a very coarse mesh of 264 elements, see Figure 6 ; a large time step $\delta t=0.1 \mathrm{~s}$ and a small one $\delta t=0.05 \mathrm{~s}$. In order to take into account the diffusive character of turbulent flows, the laminar simulations are carried out using the RANS equations with a constant eddy viscosity such that $\nu_{t}=100 \nu$. In the same way, the thermal diffusion is added a constant turbulent diffusion $\kappa_{t}=\nu_{t} / \mathrm{Pr}_{t}=4.35 \times 10^{-5} \mathrm{~m}^{2} / \mathrm{s}$. Figure 7 (Left) shows the convergence history of the iterative scheme for the turbulent solution on the fine mesh and with $\delta t=0.1$ s. The velocity residual of the equation falls 4 orders of magnitude in 10 iterations, while the eddy-viscosity residual falls less than 2 orders of magnitude. Figure 7 (Right) compares the evolution of the velocity at the inflow for four different simulations. We note that as the flow enters the plate, the front advance is pumping more and more metal; in fact, the velocity at the front is almost constant, with value

$$
u_{\mathrm{mf}} \approx \frac{\alpha_{\mathrm{mf}}}{\rho_{\mathrm{f}} c_{p \mathrm{f}}}=0.075 \mathrm{~m} / \mathrm{s}
$$



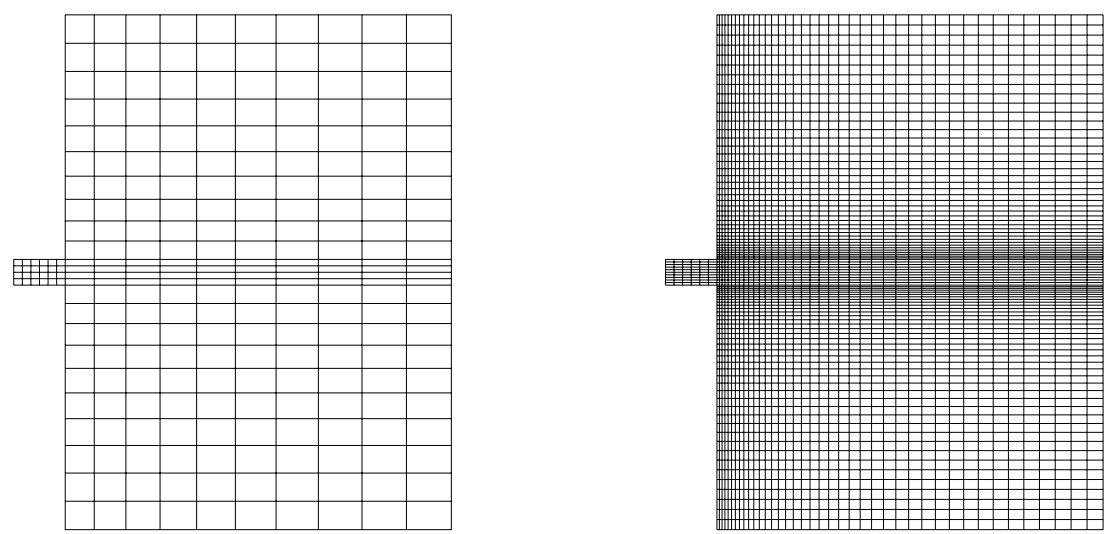

Figure 6: Horizontal plate. (Left) Coarse mesh. (Right) Fine mesh.
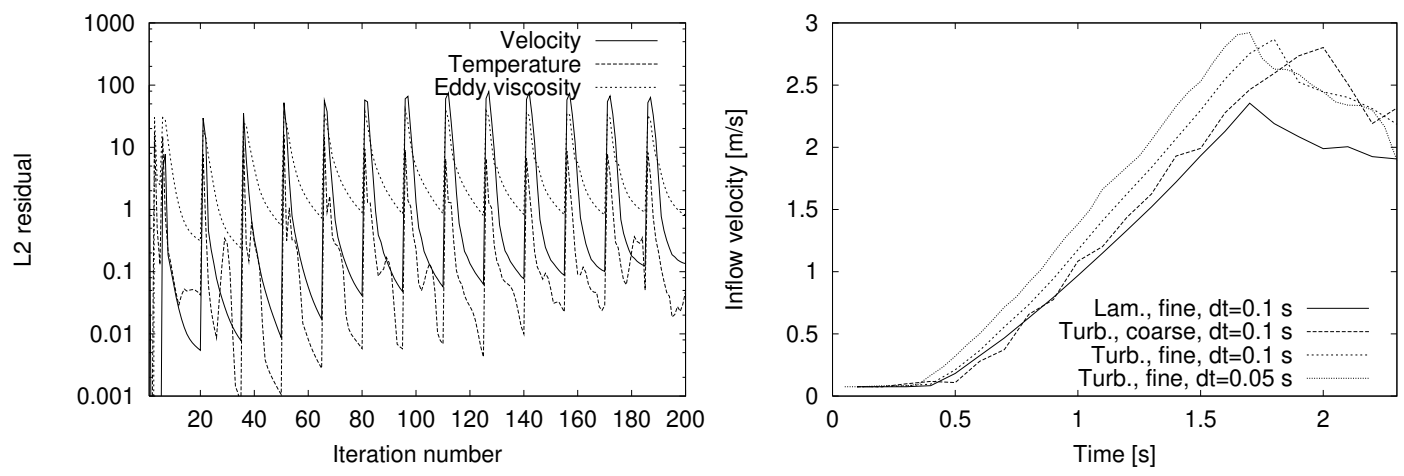

Figure 7: Horizontal plate. (Left) Convergence. (Right) Velocity at inflow.

and as the length of the front increases, the mass flow rate must increase. We observe that the laminar and turbulent solutions obtained on the fine mesh with the same time step give very similar solutions.

Figures 8 and 9 compare the evolutions of the metal front and velocity vectors for the three times shown in Figure 5. Note that for these four simulations the time is set to zero when the front passes the plate entrance. We first observe that the complicated shape of the front obtained in the experiments cannot be well reproduced, although the location of the front is good. We can also observe a strong recirculation zones generated once the metal enters the plate, and transported by the metal jet from left to right. This recirculation is even captured by the (very) coarse mesh, but located at different position. The generation of this vortex is shown in more detail in Figure (10). This figure also shows the temperature contours.

\subsection{Tee shape}

We simulate a three-dimensional tee-shaped casting, whose geometry is shown in Figure 11. We take the same physical properties as those of the previous example, and consider laminar flow (augmenting the viscosity and diffusion in the same way). The inner diameter of the vertical cylinder is $0.08 \mathrm{~m}$ while the inner diameter of the others is $0.10 \mathrm{~m}$. The geometry is symmetrical with respect to the ingate and therefore the filling should also be symmetric. However, we want to observe the effects of variable foam 

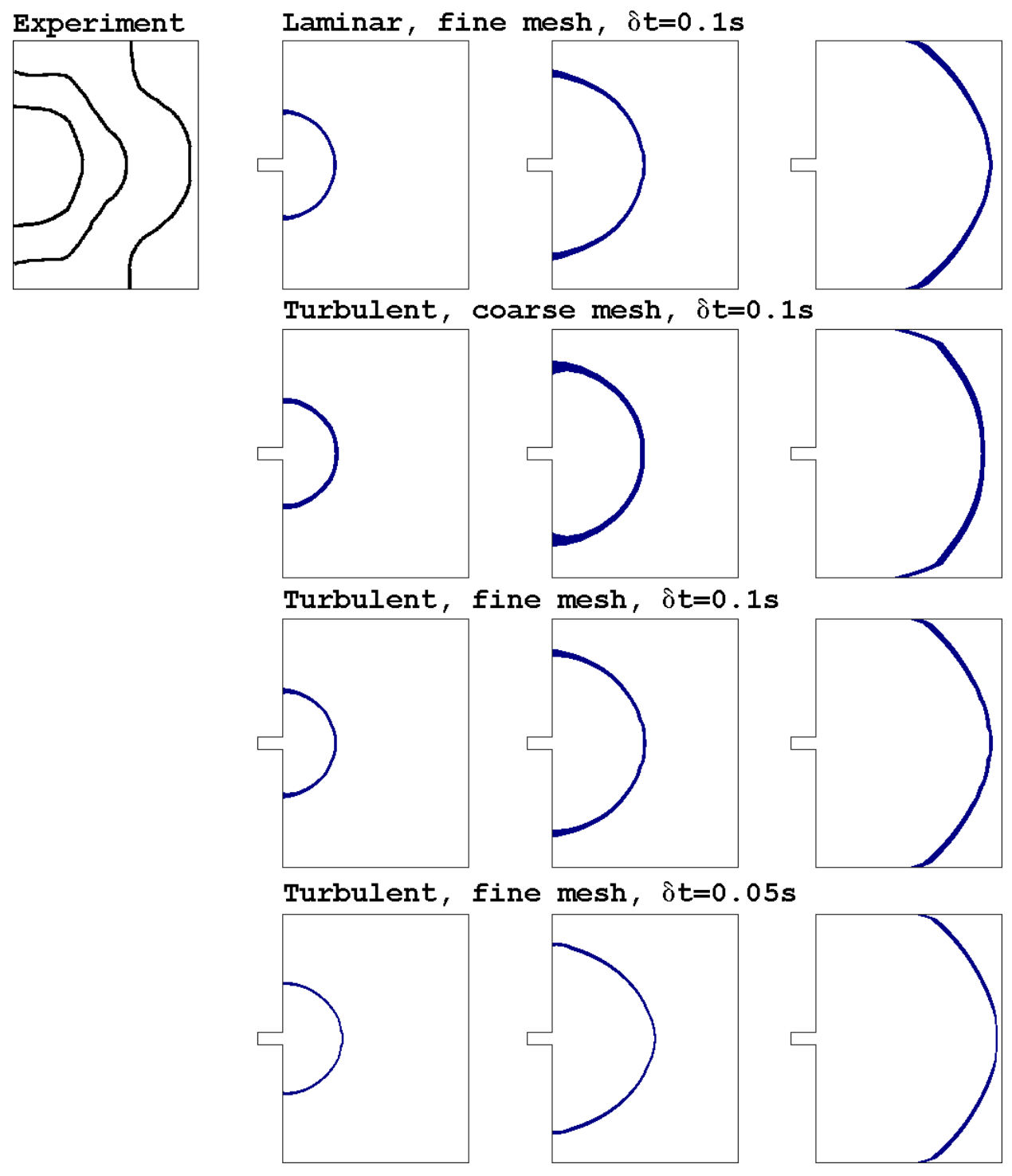

Figure 8: Horizontal plate. Front advance at time 0.6, 1.0 and $1.8 \mathrm{~s}$.

density. In fact, the foam density is likely to be non-uniform, especially near the injection points. The front velocity model given by Equation (2) is expected to take into account these effects, as the foam density appears in the denominator.

Figure (12) (Top) (Left) shows the different zones of foam density considered in this example, while Figure (12) (Top) (Right) shows the interpolated density contours on the mesh used for this simulation (25344 P1/P1 elements). On the bottom of this figure, we can observe the decrease of the metal advance velocity when it encounters zones of high density. Finally, Figure (13) shows the evolution of the velocity vectors.

\section{Conclusions}

We have presented a numerical model to solve lost foam casting problems. This numerical model is based on a finite element method using an ALE formulation with a fixed mesh. It was found that the 


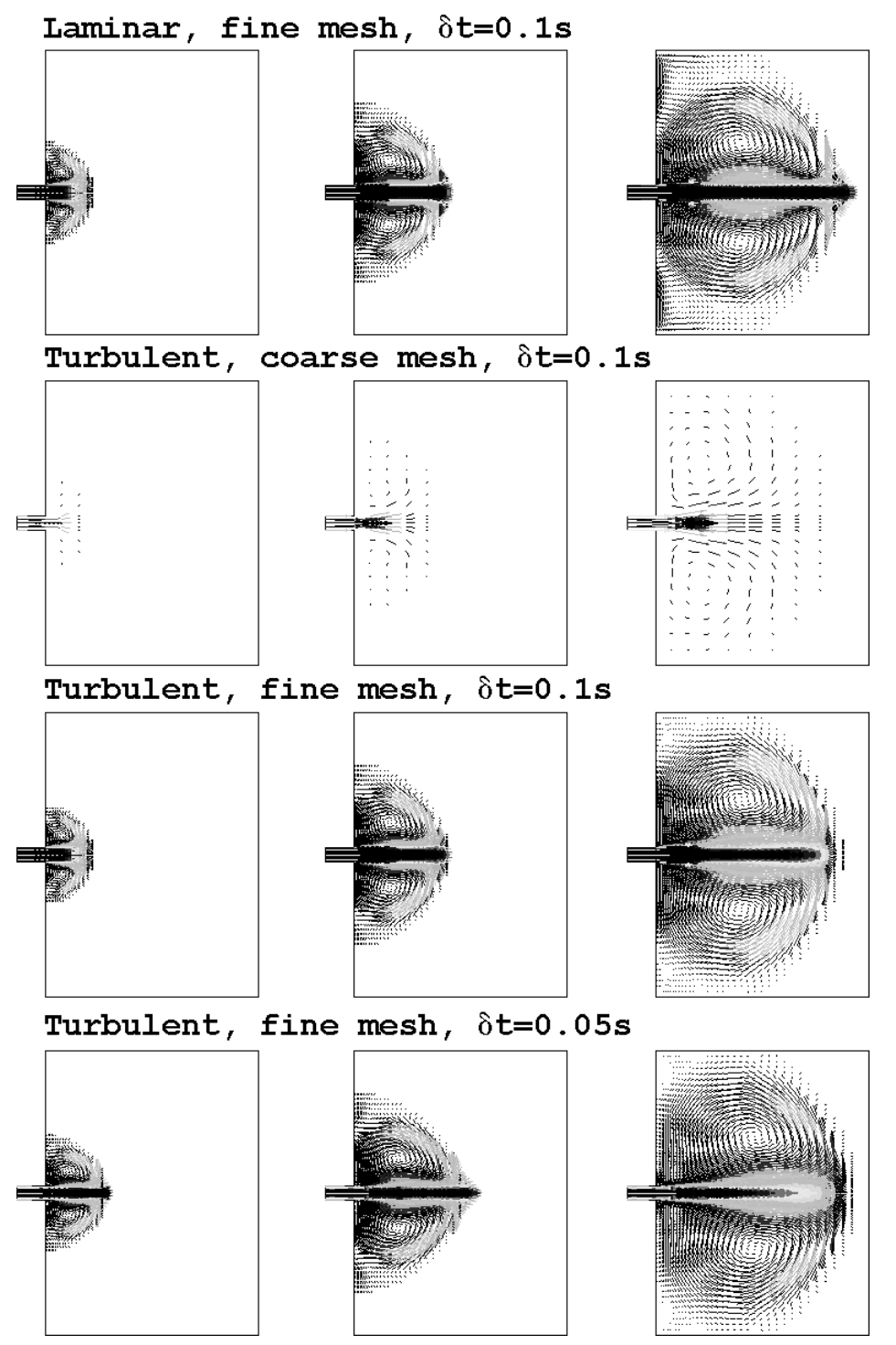

Figure 9: Horizontal plate. Velocity vectors at time $0.6,1.0$ and $1.8 \mathrm{~s}$.

evolution of the metal domain could be well captured by solving a partial differential equation for a level set function, advected on both sides of the front. The first numerical example showed that the dynamic of the metal was almost excusively driven by the front advance: the laminar simulation with augmented viscosity and the turbulent simulations gave very similar results. In the second numerical example, we showed that the model for the front velocity could take into account variable foam density. Finally, we give in the appendix some indications on how the physical model could be modified to take into account more physical processes.

\section{Appendix}

\section{A. Metal boundary reconstruction}

In section 3.2.3 we mentioned the possibility of reconstructing at each time step the metal-front boundary. This is useful if a Neumann or Robin condition is to be imposed on the temperature equation. The 


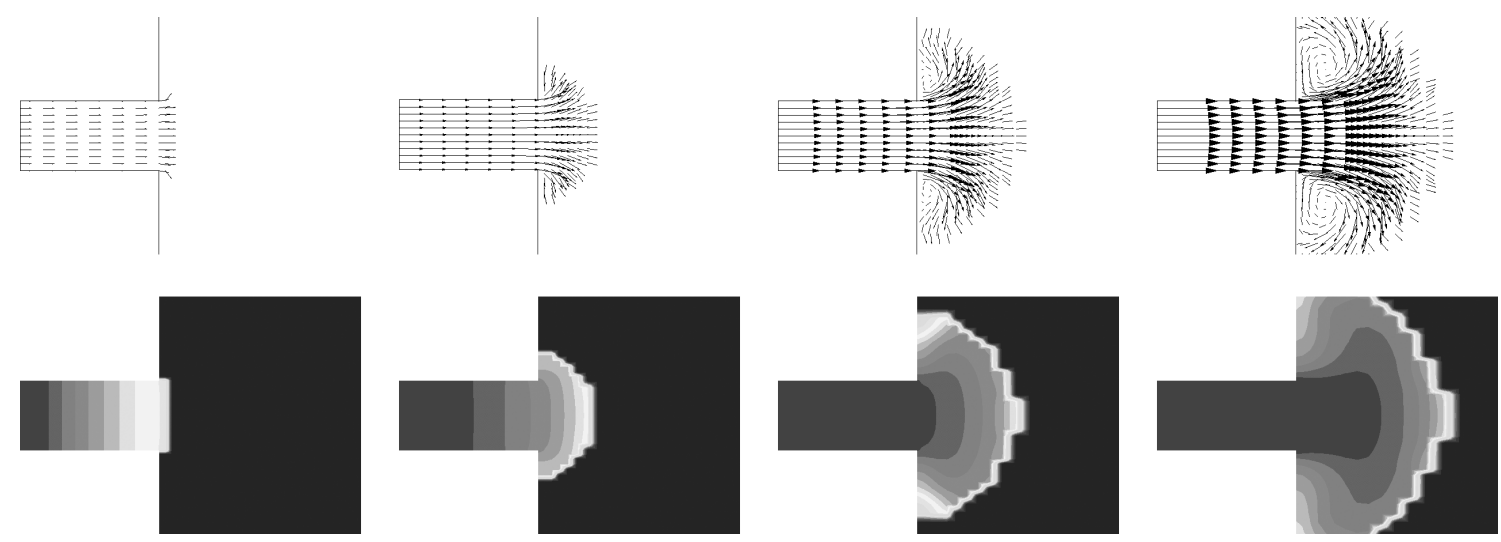

Figure 10: Horizontal plate. Metal entering the plate. (Top) Velocity vectors. (Bot.) Temperature contours.
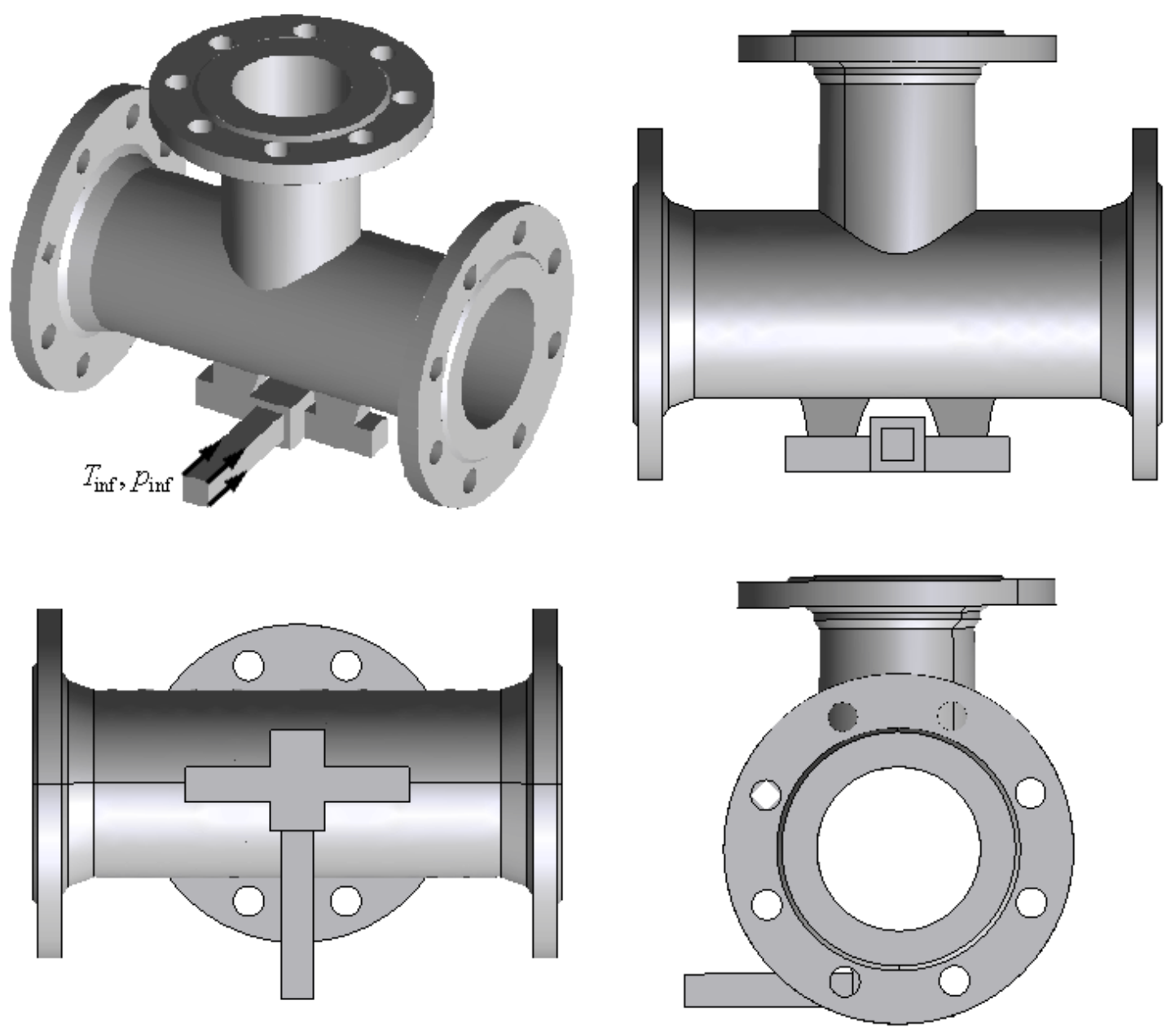

Figure 11: Tee-shape. Geometry.

algorithm to construct this boundary is given by Algorithm 2 . 

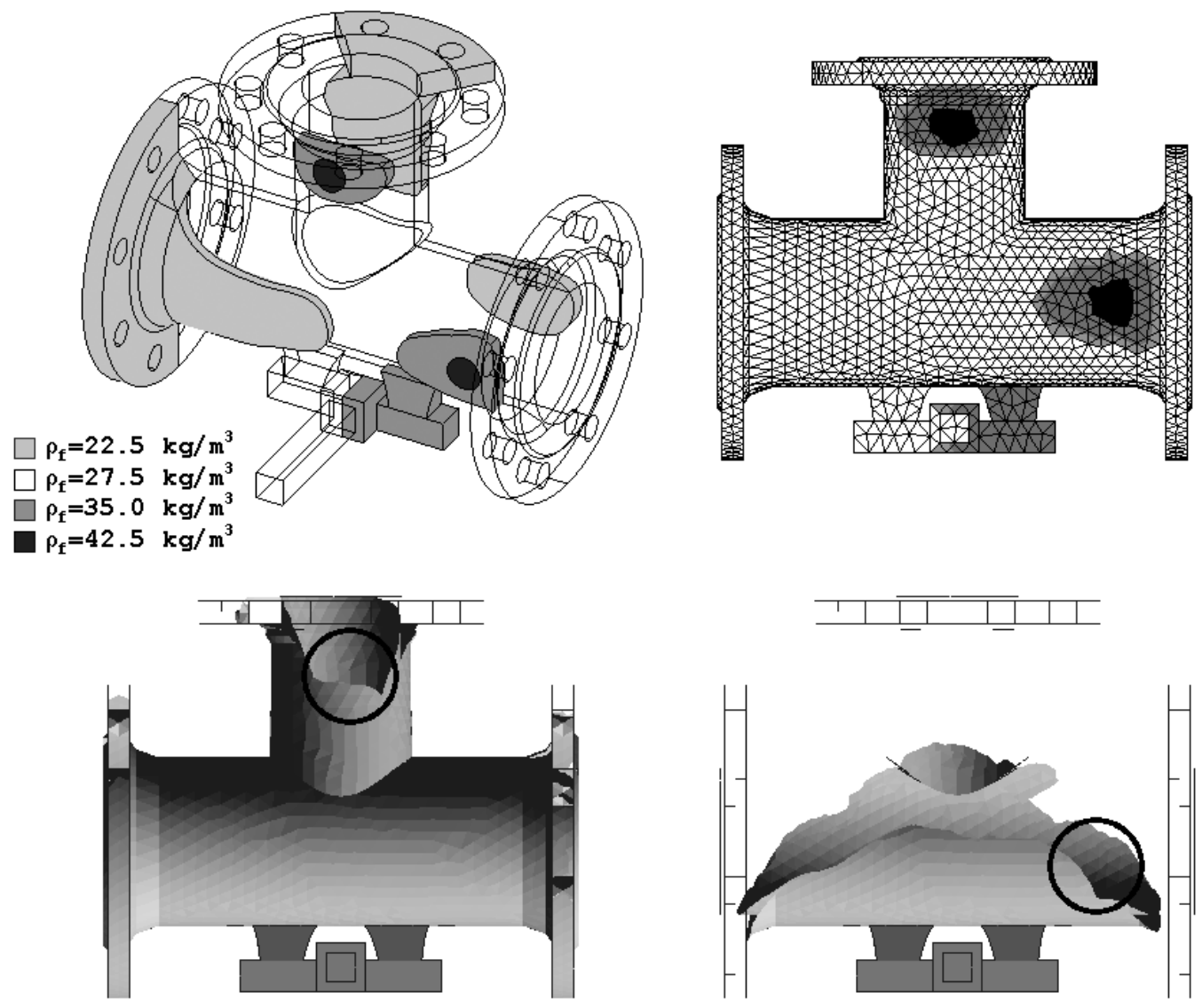

Figure 12: Tee-shape. (Top) (Left) Density zones. (Top) (Right) Mesh and density contours. (Bot.) Metal advance slown down by high foam density (indicated by the circles).

\section{B. Taking defects into account}

The prediction of defects can be achieved by solving a scalar transport equation for the concentration of residue $\eta$ of the type

$$
\begin{aligned}
\partial_{t} \eta+\boldsymbol{u}_{a} \cdot \nabla \eta & =S_{\eta} & & \text { in } \Omega_{\mathrm{m}}, \\
\eta & =0 & & \text { on } \Gamma_{\mathrm{inf}},
\end{aligned}
$$

where $S_{\eta}$ is a source term emanating from the front. As noted in [3], it can be taken proportional to the mass of degraded foam.

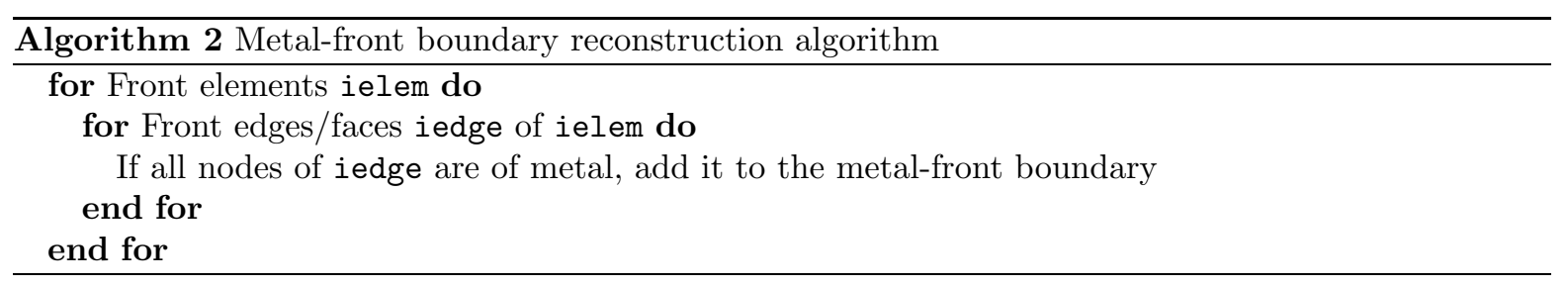



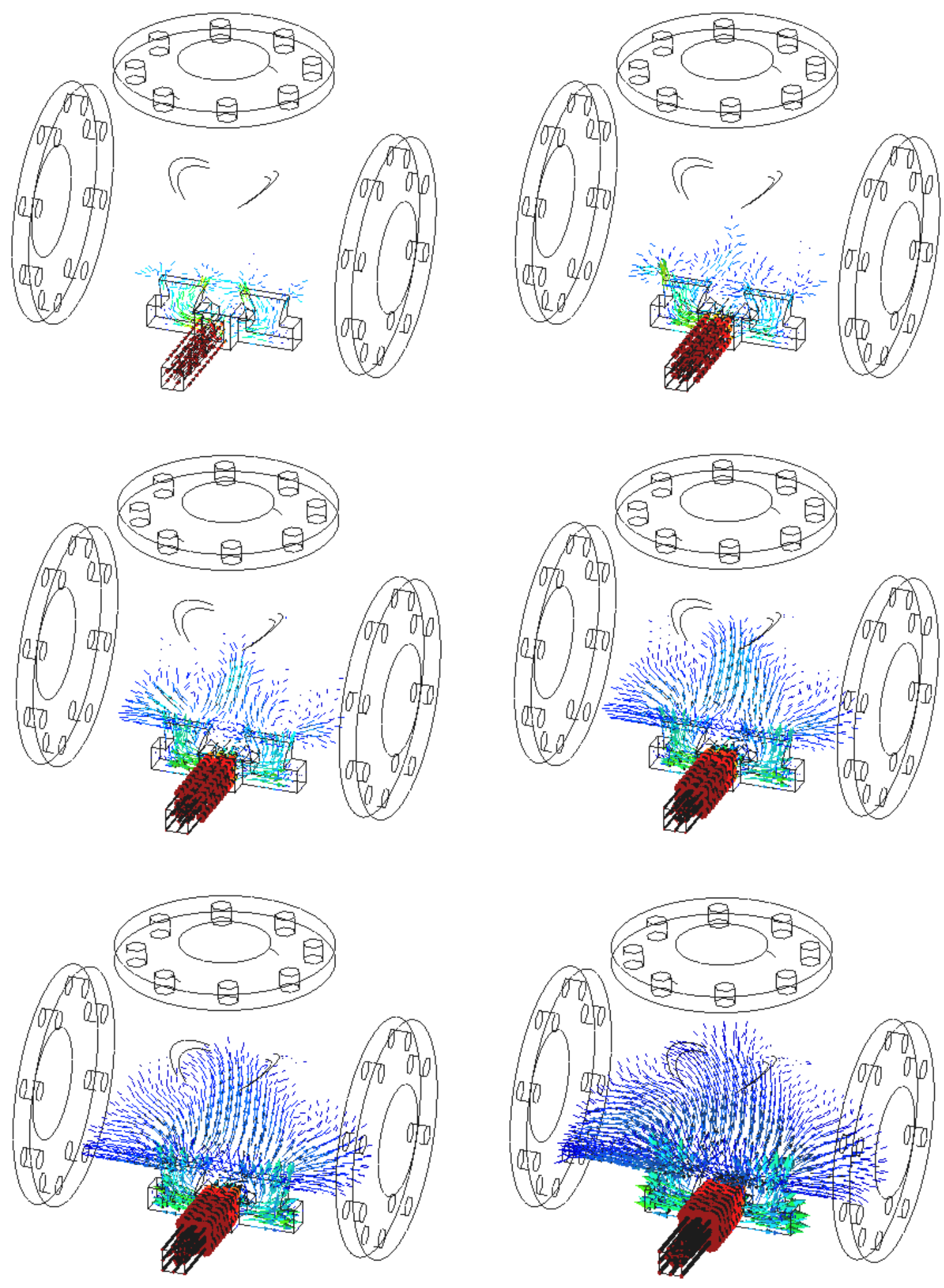

Figure 13: Tee-shape. Velocity vectors along time. 


\section{Front velocity modeling}

The model for the front advance velocity devised in this work is very simple. For example it does not take into account the effects of pouring temperature, coating, foam type, gravity, etc. We note in fact that the front velocity is independent on the mold orientation with respect to the gravity. From experiments, the front advance should depend on:

- ...the pouring temperature. The back-pressure due to the combustion of the foam increases with this temperature. For very high temperatures, the front velocity decreases with increasing pouring temperature. See for example the experiments carried out in $[22,21]$. In the first reference mentioned, filling time of the plate solved in Section 4.1 goes from 2.1s to 3.5s for temperature ranging from $490 \mathrm{C}$ to $1115 \mathrm{C}$.

- ...the coating. The type of coating determines the flow of evacuation of the gas. For high permeable coating, it is observed that the front velocity increases linearly with the pouring temperature [22]. For a given temperature, filling times can duplicate according to the type of coating used.

- ...the foam. In [23], the authors perform a multi-parameter experimental analysis to study the combined effects of foam material, coating, pouring temperature, etc. They show that the foam material is of primary importance in foam castings. In particular, they compare expendable polystyrene (EPS) and polymethyl methacrylate (PMMA). Eventhough these materials have similar densities, their chemical compositions and thermal decomposition characteristics are different so that they produce very different filling times (up to three times higher for PMMA). For example, EPS yields more viscous residue than PMMA; see Figure 1 (Right).

- ...the gravity. Although gravity has no as strong effects as in empty cavity casting, in lost foam process it can help to counterbalance the back-pressure. In [21], a filling time of $2.7 \mathrm{~s}$ is obtained for a bottom gating (counter gravity), while a filling time of $1.75 \mathrm{~s}$ is obtained for a top gating.

In addition, more drastic effects can occur. For high temperatures and low coating permeabilities, a large gap between the front and the burnt foam is observed. This situation is not envisaged in the context of this work.

We now present a simple way to take into account the effects of the back-pressure $p_{\text {gas }}$. This is the first step of model improvement. As we do not have at hand a combustion model, a formula for the backpressure must be obtained from experimental results. According to the previous remarks, we assume that

$$
p_{\text {gas }}=p_{\text {gas }}\left(T_{\text {inf }}, \text { foam, coating }\right)
$$

is a known function.

We now present an expression for the metal-to-foam heat transfer coefficient expliciting its dependence on $p_{\text {gas }}$. We assume that the heat transfer coefficient in the absence of back pressure is known, and is referred to as $\alpha_{\mathrm{mf}}^{0}$. It can be shown that the value of the heat transfer between two materials is proportional to their separation distance $d$. If we assume next that this distance is proportional to the pressure difference between the metal and the gas, then we can express the heat transfer as a function of these pressures. In particular, we have

$$
\begin{aligned}
& p=p_{\mathrm{gas}} \rightarrow d=d_{0} \rightarrow \alpha_{\mathrm{mf}}=\alpha_{\mathrm{mf}}^{0}, \\
& p>p_{\mathrm{gas}} \rightarrow d<d_{0} \rightarrow \alpha_{\mathrm{mf}}>\alpha_{\mathrm{mf}}^{0}, \\
& p<p_{\mathrm{gas}} \rightarrow d>d_{0} \rightarrow \alpha_{\mathrm{mf}}<\alpha_{\mathrm{mf}}^{0} .
\end{aligned}
$$

From these remarks, we can derive an expression for the heat transfer coefficient, similar to that used in usual thermal contact models (See [24]). We propose the following expression:

$$
\alpha_{\mathrm{mf}}:=\alpha_{\mathrm{mf}}^{0}\left(\frac{p}{p_{\text {gas }}}\right)^{\epsilon},
$$


where $\alpha_{\mathrm{mf}}^{0}$ is the heat transfer coefficient when metal and gas pressures balance each other.

This formula enables to include in a simple way the effects of the set of parameters ( $T_{\mathrm{inf}}$, coating, $\boldsymbol{g}$ ) in the determination of the front advance, as the front velocity $u_{\mathrm{mf}}$ depends explicitly on the heat transfer coefficient. Indeed, we assume that we know an expression for the gas pressure in function of the pouring temperature and the coating (Equation (10)). The dependence on the gravity appears implicitly through the dependence on the static pressure in Equation (11). We have therefore

$$
u_{\mathrm{b}}=\frac{\alpha_{\mathrm{mf}}\left(T_{\mathrm{inf}}, \text { coating, } \boldsymbol{g}\right)\left(T_{\mathrm{m}}-T_{\mathrm{f}}\right)}{\rho_{\mathrm{f}}\left[c_{p \mathrm{f}}\left(T_{\mathrm{m}}-T_{\mathrm{f}}\right)+E_{\mathrm{mel}}+E_{\mathrm{vap}}\right]} .
$$

\section{Acknowledgements}

The authors want to thank Martin Solina from Quantech ATZ for his ability for deleting the millions of lines which appeared during the importation of the CAD files. They also thank Doug Warne and Ian Almond from Saint-Gobain Pipelines for providing the tee-shape geometry. This work was carried out in the framework of the European Project FOAMCAST G1RD-CT-2000-00417.

\section{References}

[1] T.S. Piwonka. A comparison of lost pattern casting processes. Mater. Design, 11(6):283-290, 1990.

[2] L. V. Divone. U.S. industrial energy efficiency reserach, including a focus on metal casting. In NATO/CCMS Pilot Study. Clean Products and Processes (Phase I), number 238, pages 13-15. 1999.

[3] C.W. Hirt and M.R. Barkhudarov. Lost foam casting simulation with defect prediction. In B.G. Thomas and C. Beckermann, editors, Modeling of Casting, Welding and Advanced Solidification Process VIII, pages 51-57, San Diego, California (USA), June 1998. The Minerals, Metals \& Materials Society.

[4] R.W. Lewis and K. Ravindran. Finite element simulation of metal casting. Int. J. Num. Meth. Eng., 47:29-59, 2000.

[5] F. Muttin, T. Coupez, M. Bellet, and J.L. Chenot. Lagrangian finite-element analysis of timedependent viscous free-surface flow using an automatic remeshing technique application to metal casting flow. Int. J. Num. Meth. Eng., 36:2001-2015, 1993.

[6] R. Radovitzky and M. Ortiz. Lagrangian finite element analysis of Newtonian fluid flows. Int. J. Num. Meth. Eng., 43:607-619, 1998.

[7] S. Osher and R. Fedkiw. Level Set Methods and Dynamic Implicit Surfaces. Springer, 2002.

[8] R. Codina, U. Schafer, and E. Oñate. Mould filling simulation using finite elements. Int. J. Num. Meth. Heat Fluid Flow, 4(3):291-310, 1994.

[9] R.Y. Chang and W.H. Yang. Numerical simulation of mold filling in injection molding using a three-dimensional finite volume approach. Int. J. Num. Meth. Fluids, 2001.

[10] R.W. Lewis, S.E. Navti, and C. Taylor. A mixed Lagrangian-Eulerian approach to modelling fluid flow during mould filling. Int. J. Num. Meth. Fluids, 25:931-952, 1997.

[11] L. Gaston and A. Kamara an M. Bellet. An arbitrary Lagrangian-Eulerian finite element approach to non-steady state turbulent fluid flow with application to mould filling in casting. Int. J. Num. Meth. Fluids, 34:341-369, 2000. 
[12] S.R. Idelsohn, M.A. Storti, and E. O nate. Lagrangian formulations to solve free surface incompressible inviscid fluid flows. Comp. Meth. Appl. Mech. Eng., 191:583-593, 2001.

[13] P.R. Spalart and S.R. Allmaras. A one-equation turbulence model for aerodynamic flows, 1992. AIAA Paper 92-0439.

[14] C.J. Lawn. Turbulent temperature fluctuations in liquid metals. Int. J. Heat Mass Tranf., 20:10351044, 1977.

[15] M. Jischa and H.B. Rieke. About the prediction of turbulent Prandtl and Schmidt numbers from modeled transport equations. Int. J. Heat Mass Tranf., 22:1547-1555, 1979.

[16] B. Weigand, J.R. Ferguson, and M.E. Crawford. An extended Kays and Crawford turbulent Prandtl number model. Int. J. Heat Mass Tranf., 40(17):4191-4196, 1997.

[17] P.B. Bradshaw and P.G. Huang. The Law of the Wall in turbulent flows. In Proc. Roy. Soc. Lond. $A$, volume 451, pages 165-188, 1995.

[18] B. Mohammadi and O. Pironneau. Unsteady separated turbulent flows computation with wall-laws and $k-\varepsilon$ model. Comp. Meth. Appl. Mech. Eng., 148:393-405, 1997.

[19] T. J.R. Hughes. Multiscale phenomena: Green's functions, the Dirichlet-to-Neumann formulation, subgrid scale models, bubbles and the origins of stabilized methods. Comp. Meth. Appl. Mech. Eng., 127:387-401, 1995.

[20] R. Codina. A stabilized finite element method for generalized stationary incompressible flows. Comp. Meth. Appl. Mech. Eng., 190, 2001.

[21] X. Yao and S. Shivkumar. Mold filling and solidification in the lost foam process. afs 95-116. In 99th AFS Casting Congress, pages 761-765, Kansas City, Missouri (USA), April 1995.

[22] X. Liu, C.W. Ramsay, and D.R. Askeland. Study on mold filling control mechanisms in the epc process. afs 94-148. In 98th AFS Casting Congress, pages 903-914, Hamilton, Ontario (Canada), May 1994.

[23] C. Wang, C.W. Ramsay, and D.R. Askeland. Effect of processing parameters on mold filling for gray iron epc castings using statistical experimental techniques. afs 94-151. In 98th AFS Casting Congress, pages 921-930, Hamilton, Ontario (Canada), May 1994.

[24] C. Agelet de Saracibar. Numerical analysis of coupled thermomechanical frictional contact problems. Computational model and applications. Arch. Comp. Meth. Eng., 5(3):243-301, 1998. 